\title{
SEVERE PANTON VALENTINE LEUKOCIDIN METICILLIN-SENSITIVE STAPHYLOCOCCUS AUREUS INFECTION: A CASE REPORT
}

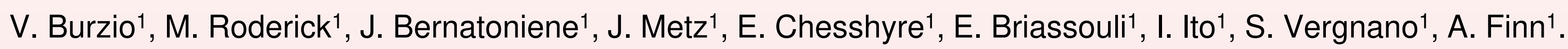

${ }^{1}$ Bristol Children Hospital, Paediatric Infectious Diseases and Immunology, Bristol, United Kingdom

\section{Background}

Panton Valentine Leukocidin Staphylococcus aureus (PVL$\mathrm{SA})$ has repeatedly been associated with more severe disease than PVL negative $S$. aureus infections, although controversy exists as to whether PVL-toxin is the major virulence determinant ${ }^{1}$.

\section{Case Presentation}

A previously well two-yearsold girl presented in poor clinical conditions to the emergency department with a 5 days history of fever and swollen left eye with mildly reduced abduction. The inflammatory markers were high (CRP $224 \mathrm{mg} / \mathrm{l})$. She was diagnosed with periorbital cellulitis and was started on intravenous ceftriaxone and metronidazole.
A brain MRI confirmed the diagnosis. Her blood culture grew fully sensitive $S$. aureus after one day. Therapy was switched to flucloxacillin, and when PVL was reported, rifampicin was added. A cardiac ultrasound revealed no abnormalities. However, she developed a full sixth nerve palsy after five days of treatment and an urgent brain CT showed a small extra-axial collection in the posterior fossa, and septic emboli in both lungs (figure below).

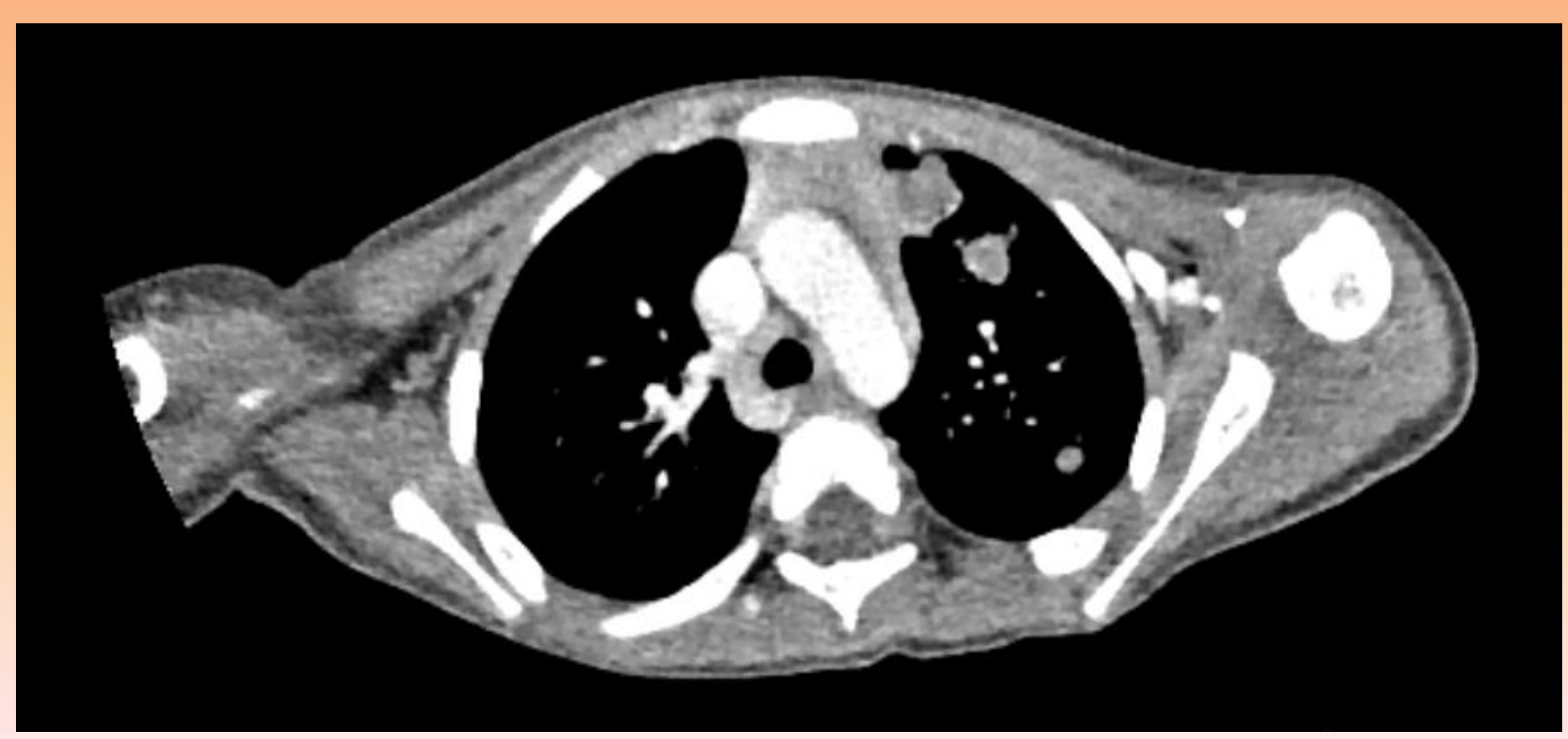

Repeated brain MRI reported a cavernous sinus thrombosis. Once stable, using an elastomeric device for flucloxacillin delivery, the patient was discharged under the supervision of our paediatric outpatient parenteral antimicrobial therapy team (p-OPAT). She required a prolonged intravenous treatment course of 3 months and completed her antimicrobial treatment with a further month of oral amoxicillin-clavulanate.
1 Boan et al. BMC Infectious Diseases (2015) 15:10
The patient made a full recovery, without any ophthalmology or audiology complications. Neutrophil function, lymphocytes subsets and immunoglobulins resulted normal.

\section{Discussion}

This case illustrates that PVLMSSA can cause very severe invasive disease in children even with normal immune system. Clinicians and laboratories should routinely submit isolates for PVL from children with invasive MSSA disease, because this would improve the knowledge of the epidemiology of the disease. Optimal antimicrobial combination and length of therapy is not well established.

University Hospitals Bristol W/HS NHS Foundation Trust 